\title{
Futuro de la enseñanza médica: inteligencia artificial y big data
}

\author{
Óscar García Esquirol
}

Hospital de Sant Joan Desp Moisès Broggi.

(c) 2015 FEM

Resumen. La medicina digital se presenta como una solución a los problemas asistenciales actuales. En este contexto nace Teckel Medical, una empresa dedicada al desarrollo de software médico basado en inteligencia artificial. Su aplicación Mediktor es el primer evaluador de síntomas avanzado del mundo, capaz de reconocer lenguaje natural para que el usuario exprese cómo se siente con sus palabras. Conduce un interrogatorio médico hasta concluir en un listado de posibles enfermedades asociadas a los síntomas referidos. La inteligencia artificial de Mediktor, junto con técnicas de gamificación, pueden utilizarse en el campo de la formación para dinamizar la enseñanza. La Universidad de Barcelona, el Hospital Clínic y Mediktor se unen para desarrollar una herramienta de formación, información y evaluación destinada a estudiantes de medicina. El objetivo es crear una herramienta de enseñanza atractiva que fidelice su uso por parte del estudiante. La revolución en formación médica ha empezado.

\section{Future of medical education: artificial intelligence and big data}

Summary. Digital medicine can be a solution to current health care problems. This is the setting in which Teckel Medical was born - a company devoted to the development of medical software based on artificial intelligence (Al). Its Mediktor app is the world's first advanced symptom evaluator, capable of recognizing natural language so that users can express themselves in their own words. It runs through a medical query session until it concludes with a list of possible diseases associated with the reported symptoms. Mediktor's Al, together with gamification techniques, can be used in education in order to enhance teaching. Universitat de Barcelona-Hospital Clínic and Mediktor have joined forces to develop a training, information and evaluation tool aimed at medical students. The goal is to create an attractive teaching tool that encourages its use by the student. The revolution in medical training has begun.

\section{Medicina, un antes y un después...}

Hablar de medicina del futuro está de moda. Aparecen términos de diseño ('mHealth', 'eHealth', 'wearables', etc.) que buscan dar nombre a una obviedad: momento en el que coexisten una necesidad médica por la escasez de recursos y una revolución tecnológica que puede ser su solución. Tener un ordenador de bolsillo de poco más de 100 gramos con una capacidad de procesamiento cinco veces superior a la que tenía el megaordenador de IBM que envió al ser humano a la Luna lo ha cambiado todo.

Los recursos son limitados y no cubren las necesidades de una población cada vez más añosa y con patologías más crónicas. En el momento de sacar músculo ocurre todo lo contario, afloran las defi- ciencias de un sistema sanitario obsoleto. Se ponen de manifiesto desigualdades sociales y culturales que sólo pueden equilibrarse con una solución eficiente, medicina digital, 'buena bonita y barata' La medicina del siglo XXI está aquí nos guste o no, y ha llegado para quedarse.

En este marco aparece Mediktor, la aplicación de referencia para el diagnóstico médico. Considerada el primer evaluador de síntomas del mundo capaz de reconocer lenguaje natural, para que el usuario exprese con sus palabras cómo se siente y qué síntomas presenta. Utiliza inteligencia artificial (IA) para pensar como lo haría un médico y conducir un interrogatorio hasta llegar a un listado de diagnósticos posibles. Según las enfermedades, recomienda el especialista al que se ha de consultar, permitiendo 
chatear en tiempo real con un médico para recibir consejo personalizado. El médico ayuda a tomar la mejor decisión sobre el estado de salud del usuario.

\section{La medicina 2.0 Ilega a las aulas}

Los formadores de la salud entienden que existe un momento de cambio, por lo que se empieza a enseñar medicina 2.0 en las facultades, preparando a los futuros profesionales para responder a las nuevas preferencias de los usuarios.

Hasta hace unas décadas la formación médica era lineal, en un solo plano. Clases magistrales impartidas por 'sabios' que transmitían conocimiento sin que hubiera una medicina basada en la evidencia, sino más bien una medicina basada en su experiencia. Los alumnos recibían la información en un solo canal, con escasa interacción, lo que dificultaba aclarar dudas y conceptos.

Con el tiempo la formación se convierte en una enseñanza en dos planos: teoría monotemática y prácticas que asientan los conceptos adquiridos. Aparecen los primeros guiños tecnológicos: aulas de simulación, plataformas Moodle, bibliotecas de señales biomédicas, etc. Se transmiten conocimientos de cardiología, de neumología, de patología digestiva, etc., pero siempre de una manera compartimentada, parcial, sin relacionar unos sistemas con otros.

Con el siglo Xxi llega la formación en 3D, donde dos entidades cambian la manera de estudiar medicina: IA y gamificación. Al combinar máquinas que piensan como los humanos y el empleo de mecánicas de juego que potencian la motivación, la concentración y la fidelización, se crea una herramienta muy atractiva para el estudiante.

La IA permite una enseñanza dinámica, relacional, sin paredes. Utilizando una serie de algoritmos que dirigen los árboles de decisión, se puede llegar a una serie de enfermedades partiendo de un síntoma guía o totalmente al revés, llegar a un grupo de síntomas partiendo de una enfermedad. Las combinaciones son infinitas y configurables a gusto del formador. En este contexto, la Universidad de Barcelona se pone en contacto con Mediktor para utilizar su IA y su base de datos con el objetivo de desarrollar una herramienta puntera de formación. ¿Es

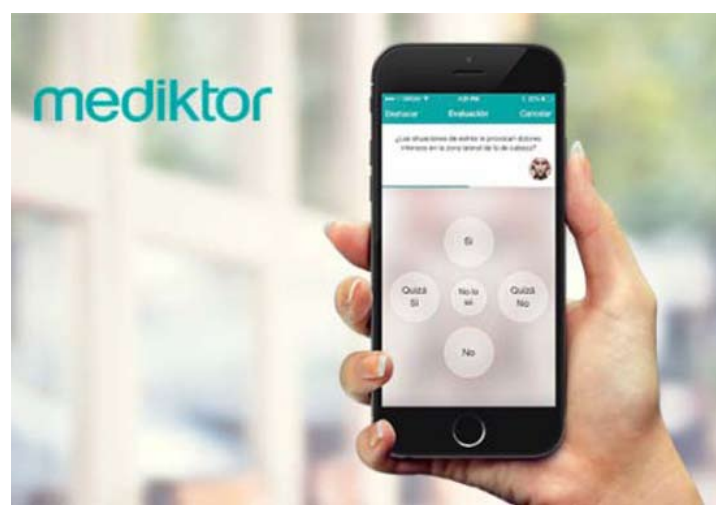

posible invertir las preguntas de la aplicación para que el estudiante realice una anamnesis y Mediktor le conteste?

El uso de la gamificación está cada vez más extendido en todos los ámbitos. En formación se ha demostrado que 'aprender jugando' es una herramienta muy potente para asentar conocimientos. Probablemente si a los adolescentes de hoy les pedimos que citen cinco elementos de la tabla periódica estarán en apuros, pero si les pedimos que nombren cinco personajes de Pokémon responderán en segundos. Los conocimientos no entran con sangre, entran generando interés.

Cambiar el porcentaje de minutos destinados al ocio en las redes sociales, a las ventas online, etc., por minutos de formación ahora es posible. Generemos interés, los ingredientes están en nuestra mano: smartphones, IA y gamificación. El estudiante encontrará un pasatiempo enriquecedor y una plataforma para conectar con otros estudiantes y generar foros de debate. El uso de esta herramienta creará una gran base de datos (big data) que permitirá al formador modular temarios, foros de discusión y casos prácticos. Tendrá una herramienta de evaluación continua que le permitirá testar las habilidades médicas de los usuarios de una forma muy práctica y parecida a los casos reales. El resultado final será una enseñanza viva, dinámica y global, donde se relacionarán conocimientos de los diferentes sistemas y órganos para estudiar el organismo como un todo. El reto es importante, y los objetivos, prometedores. La revolución de la formación médica ha empezado. 